\title{
Éxito universitario en el País Vasco: el programa Arrakasta
}

\author{
Miguelena Torrado, Joana \\ Universidad del País Vasco/Euskal Herriko Unibertsitatea UPV/EHU, Donostia, España \\ joana.miguelena@ehu.eus
}

Dávila Balsera, Paulí

Universidad del País Vasco/Euskal Herriko Unibertsitatea UPV/EHU, Donostia, España

pauli.davila@ehu.eus

Naya Garmendia, Luis María
Universidad del País Vasco/Euskal Herriko Unibertsitatea UPV/EHU, Donostia, España
luisma.naya@ehu.eus

\section{Resumen}

Con motivo de una investigación sobre el derecho a la educación de las niñas, niños y adolescentes atendidos bajo una medida de protección de acogimiento residencial en la Diputación Foral de Gipuzkoa, se detectó la dificultad que tenía este colectivo para acceder y mantenerse dentro del sistema de educación superior. Con el objetivo de implementar las condiciones que permitan esta forma exitosa de acceder y mantenerse en la universidad, la Universidad del País Vasco y la Diputación de Gipuzkoa proyectaron el programa Arrakasta (éxito en euskara). Estas instituciones resolvieron favorablemente la propuesta, comprometiéndose a crear y mantener recursos, tanto institucionales como económicos a fin de garantizar el éxito de este colectivo en los estudios universitarios. En el curso 2017-18 ha sido firmado el acuerdo interinstitucional y puesto en marcha el programa. En este proyecto se ha involucrado la Fundación SM para coadyuvar económicamente mediante becas y aportaciones financieras a fin de garantizar el éxito en el mismo.

Las primeras impresiones de jóvenes que están participando en el programa Arrakasta acerca de los beneficios de éste en su itinerario universitario nos muestran, entre otros, los siguientes beneficios: visibilidad del colectivo, seguridad en el seguimiento de estudios superiores, orientación y apoyo universitario, obtención de infraestructura a cargo de la universidad, apoyo económico, implicación de instituciones ajenas a la universidad, etc.

Al margen de estas cuestiones de tipo práctico, y en relación más directa con los resultados de la investigación, hemos podido constatar que los profesionales que trabajan en este ámbito ampliarían la visión sobre los horizontes académicos de este colectivo, facilitando información de las oportunidades que pueden tener en el ámbito de la educación superior, además del cambio de expectativas de este colectivo que pueden ver también oportunidades de estudio en la universidad.

Arrakasta es un programa pionero y que puede servir de modelo para que otras instituciones en una situación similar, puedan aplicar este tipo de prácticas exitosas en la educación superior.

Palabras clave: Educación superior, igualdad de oportunidades, éxito escolar, País Vasco, colaboración institucional, inclusión.

\section{INTRODUCCIÓN}

Con motivo de una investigación sobre el derecho a la educación de niñas, niños y adolescentes (NNA) atendidos bajo una medida de protección de acogimiento residencial en la Diputación Foral de Gipuzkoa (DFG), se detectó la dificultad que tenía este colectivo para acceder y mantenerse dentro del sistema de educación superior. Con el objetivo de implementar las condiciones que permitan esta forma exitosa de acceder y mantenerse en la universidad, la Universidad del País Vasco y la Diputación de Gipuzkoa proyectaron el programa Arrakasta (éxito en euskara). Estas instituciones resolvieron favorablemente la propuesta, comprometiéndose a crear y mantener recursos, tanto institucionales como económicos a fin de garantizar el éxito de este colectivo en los estudios universitarios. En el curso 2017-18 ha sido firmado el acuerdo interinstitucional y puesto en marcha el programa. En este proyecto se ha involucrado la Fundación SM para coadyuvar económicamente mediante becas y aportaciones financieras a fin de garantizar el éxito en el mismo. 


\section{El derecho a la educación y el Derecho a la educación superior}

Desde hace décadas, los organismos internacionales han considerado el Derecho a la Educación como la base para poder tener acceso al resto de Derechos Humanos. Siguiendo «el esquema de las 4 Aes» de Katarina Tomaševski $(2004,2005)$, la protección del Derecho a la Educación supondrá la posibilidad de lograr una educación asequible, accesible, aceptable y adaptable.

El Derecho a la Educación funciona como un multiplicador, potenciando todos los derechos y libertades siempre que esté garantizado (Tomaševski, 2006), proporcionando el control a la persona sobre el curso de su vida y facilitando su participación en la vida política, económica, social y cultural (Beiter, 2005).

Según el artículo 26 de la Declaración Universal de Derechos Humanos «el acceso a los estudios superiores será igual para todos, en función de los méritos respectivos». Los Estados, de acuerdo con el artículo 4 la Convención de la UNESCO relativa a la lucha contra la discriminación en la esfera de la enseñanza (1960), deben «hacer accesible a todos, en condiciones de igualdad total y según la capacidad de cada uno, la enseñanza superior». Al hilo de lo anterior, la UNESCO (1988) también hacía referencia a este principio de no discriminación e igualdad de oportunidad de acceso a la educación superior, argumentado que «en el acceso a la educación superior no se podrá admitir ninguna discriminación fundada en la raza, el sexo, el idioma, la religión o en consideraciones económicas, culturales o sociales, ni en incapacidades físicas».

La exención de tasas en los estudios universitarios es una de las medidas que contemplan los distintos Estados para paliar desigualdades de determinados colectivos y que éstos tengan las mismas oportunidades que el resto del alumnado para realizar y completar su itinerario educativo, abogando así por ese principio de no discriminación. Centrándonos en nuestro territorio, el País Vasco, los colectivos exentos de tasas universitarias son las familias numerosas clasificadas en la categoría especial, reducción del $50 \%$, de los precios públicos por servicios académicos universitarios cuando sean miembros de familias numerosas clasificadas en la categoría general, víctimas de terrorismo y familiar, familias que tengan alguna persona con discapacidad y víctimas de violencia de género1. Como se puede constatar, las personas procedentes del sistema de protección social a la infancia y la adolescencia no están contemplados, aun, siendo un colectivo formado por jóvenes que se ven abocados a dejar los recursos residenciales del sistema de protección al cumplir los 18 años y afrontar un tránsito a la vida adulta, cargado de complejidades (Melendro et al. 2016).

En los últimos años, los estudios sobre el ámbito educativo de NNA atendidos en el sistema de protección y de los itinerarios educativos de jóvenes que egresaron de éste, han aportado luz a un problema hasta entonces no contemplado (Montserrat y Casas, 2010; Casas y Montserrat, 2012). Por distintas investigaciones sabemos que suelen presentar peores resultados en el ámbito académico que la población general (Panchón, 1999; Montserrat et al. 2011; Montserrat, Casas y Baena, 2015). No obstante, el acceso a la educación superior constituye un claro factor protector para la vida de esta población (Jackson y Martin, 1998; y Mallon, 2007). Pero, ¿cuántos jóvenes procedentes del sistema de protección suelen llegar a la universidad?

Según Montserrat et al (2011) en Reino Unido, un 9\% de los ex tutelados asistía a la universidad y un 45\% participaba en algún tipo de educación post obligatoria (este porcentaje es muy superior al que tenía Reino Unido en 2003 donde únicamente accedía a la universidad uno de cada cien estaba matriculado en programas de titulación universitaria. Este cambio se ha dado por el destacado progreso que ha impulsado este país en relación a la educación del colectivo de jóvenes procedentes del sistema de protección). Los últimos datos hechos públicos por el Ministerio de Educación apuntaban que un 14,3\% de los ex tutelados cursaban estudios universitarios, mientras que la tasa neta de escolarización universitaria de la población en general de entre 18 y 24 años es del $28,6 \% 2$. No obstante, el porcentaje ofrecido por el Ministerio de Educación nos parece alto, en comparación con los datos ofrecidos en otras investigaciones, donde el porcentaje de jóvenes extutelados en la universidad sería más bajo; un 4\% (FEPA, 2017); un 5\% (ASJTET,

\footnotetext{
1 Para más información véase también la ORDEN de 20 de junio de 2017, de la Consejera de Educación, por la que se fijan los precios a satisfacer por los servicios públicos de educación superior de la Universidad del País Vasco / Euskal Herriko Unibertsitatea para el año académico 2017-2018 y se definen las condiciones para el beneficio de las exenciones y reducciones de los mismos. Recuperado de https:// www.euskadi.eus/y22-bopv/es/bopv2/datos/2017/06/1703318a.pdf.

$2 \mathrm{https}: / /$ valenciaplaza.com/los-extutelados-por-la-generalitat-quedaran-exentos-de-tasas-universitarias.
} 
2008 citado en Montserrat y Casas, 2010); 7,5\% (Cashmore, Paxman y Townsend, 2007). De hecho, en investigaciones realizadas sobre los itinerarios formativos de jóvenes procedentes del sistema de protección, siempre ha quedado constancia de que la tasa de jóvenes extutelados que acceden a la universidad es considerablemente baja (Harvey et al. 2015; Jackson y Martin, 2002; Montserrat et al. 2011). Distintas autoras y autores han indicado que para que el colectivo de jóvenes extutelados pueda cursar sus estudios superiores necesitar seguir contando con apoyo financiero, práctico y emocional (Jackson y Martin, 2002), así como de una reforma en las políticas educativas y comunitarias, y un cambio de mirada en las expectativas depositadas en este colectivo. Entre las reformas educativas que contemplan Harvey et al. estaría, entre otras, la exención de tasas de este colectivo (Harvey et al. 2015).

Por su parte, Montserrat y su equipo proponen algunos factores facilitadores para que estas y estos jóvenes puedan continuar sus estudios tras la salida del sistema de protección. Entre ellos indican: la estabilidad de un adulto de referencia; que el servicio que presta el apoyo conceda prioridad al tema escolar; que se les proporcione orientación escolar que promueva los estudios postobligatorios, de acuerdo con sus preferencias y aspiraciones de futuro; la alta implicación y las elevadas expectativas de la persona guardadora en el tema formativo; la transmisión del valor de los estudios por parte del guardador y del profesorado; la implicación del instituto para que el joven no abandone la educación dadas sus circunstancias de dificultad; la existencia de servicios de ayuda para la vivienda, de becas para seguir estudiando y de acompañamiento personalizado para quienes lo necesiten, que mitigue el miedo y la inseguridad que les produce cumplir la mayoría de edad sin tener apoyo familiar (Montserrat et al. 2011, pp. 236-238).

\section{Punto de inicio: una detección casual}

La detección del «problema» se sitúa en el contexto de una investigación, enmarcada en una tesis doctoral que estamos llevando a cabo en el grupo de investigación de Estudios Históricos y Comparados en Educación-Garaian de la UPV/EHU, financiada por la Universidad del País Vasco/Euskal Herriko Unibertsitatea (UPV/EHU).

El objetivo principal de esta investigación es analizar la incidencia de la representación social que las y los profesionales que trabajan en acogimiento residencial tiene en los derechos de estas NNA. Aunque consideramos que los derechos de las NNA son indivisibles e interdependientes (Dávila y Naya, 2011), quisimos poner el foco principalmente en los derechos de participación y educación.

De acuerdo con el objetivo general de la investigación, nos adentramos en un trabajo de campo a través de cuestionarios a profesionales, entrevistas semi-estructuradas a 15 profesionales y a 15 jóvenes que habían llegado a la mayoría de edad atendidos en un recurso residencial de protección.

La detección fue casual, pero se fue revelando paulatinamente, en el transcurso del análisis de las entrevistas de profesionales y jóvenes y, sobre todo, por la relación personal continuada (fuera de la investigación) con las y los jóvenes.

\subsection{La universidad no es para las NNA atendidas en AR}

Tanto en las entrevistas de profesionales como de jóvenes se pudo percibir que la universidad no era un itinerario educativo al que solían dirigirse las NNA atendidas en AR. Los itinerarios habituales de estas NNA eran "ESO, pero en general, en general, en general, casi todo el mundo acaba en un $P C P I »$ (profesional, mujer), aunque también se mencionan casos e itinerarios no tan habituales como "grado medio ha habido alguna también» (profesional, mujer) o "tenemos algún caso que ha estado en bachillerato» (profesional, hombre). Estos mismos itinerarios son mencionados por las y los jóvenes entrevistados,

"yo era la única que estaba en casa haciendo bachiller. Los demás, CIPs, un compañero la ESO, que estaba en el mismo piso que yo, pero eso, todos los demás eran ... CIPs» (joven, mujer).

En algunas entrevistas de las y los profesionales, se hizo mención a casos «no habituales» «excepcionales» de adolescentes que habiendo estado tutelados habían llegado a la universidad. En palabras de una profesional, "yo llevo aquí más de diez años y acceso a la universidad he visto sólo en uno de ellos» (profesional, mujer)». 
El acceso a la universidad de NNA procedentes del sistema de protección no es mencionado entre las y los jóvenes. Únicamente hicieron alusión a la universidad, las jóvenes que estaban cursando en el momento de la entrevista un grado universitario, o jóvenes que querían matricularse en la universidad en el siguiente curso académico.

Cuando se les planteó la pregunta a las y los profesionales acerca de si se les solía animar a cursar estudios universitarios, hubo quien contestó que «si ese es su sueño no vamos a cortárselo porque esa también es una motivación de cara a poder seguir estudiando y poder ir hacia adelante» (profesional, hombre), pero la mayoría de las respuestas giraron en torno a la frase de "hay que ser realista». Rescatando algunas citas de las entrevistas de profesionales,

«umm no. Siempre se intenta en todos los ámbitos, se intenta individualizar, a nosotros cuando nos viene un chaval se intenta individualizar, trabajar individualmente, ver qué es lo que, con qué viene, qué es lo tiene y adonde lo queremos dirigir. Lo que no vamos a hacer nunca es intentar enfocar a los chavales a la universidad porque no va a ser real, porque luego digamos la ostia que se va a dar es mayor. Entonces tenemos que intentar ser realistas con las capacidades de cada chaval y conseguir los objetivos a corto-largo plazo pero que sean reales» (profesional, hombre)

«ellos al final también se ponen igual unas metas que igual las tienes que bajar, ¿no? igual tienes que empezar con unas metas más bajas, más básicas, y de ahí ir tirando, porque a la universidad... si la universidad es realmente es lo que quieres, con 25 años puedes entrar, o sea, al final tienen miles de formas de entrar. Ahora vamos a ser también un poco realistas, vamos a ver lo que tienes y a lo que podemos llegar, no puedes empezar por el tejado, hay muchos...» (profesional, hombre)

«me pesa que a los chavales el discurso que se les dé es: no creo que vayas a hacer bachiller o no creo que vayas a hacer... tienes que ser realista, no vas a llegar a la universidad. Esto se escucha muchísimo, desde el piso también sí, sí. (profesional, mujer)

Esta argumentación de ser «realista» podría estar influenciada por la salida de las y los adolescentes a los 18 años de edad, ya que, como comentaba una profesional:

«los 18 años es un límite con el que ellos cuentan y con el que nosotros contamos, entonces, al final, lo que nos hace es acelerar determinados procesos o determinadas situaciones que en la población normal no es así» (profesional, mujer)

Las dificultades para hacer frente a los gastos que ocasionan los estudios universitarios por la falta de apoyos económicos también son mencionadas por profesionales como uno de los motivos por los cuales no van a poder realizar estudios universitarios.

«llegan a los 18 años eh... puff... voy a la universidad, la universidad hay que pagarla, hay que... si no tienes quien te sostenga, su peor problema es la red social que les rodea» (profesional, mujer)

«pero porque las características de haber pasado por un piso... (...) a lo mejor no puedes acceder a una universidad, porque ya el sistema te lo va a hacer muy difícil para acceder. A lo mejor tú querías ir a la universidad, pero sabes que tu situación no te lo permite y ya es una cosa que te va a influir en el resto de tu vida» (profesional, mujer)

Los testimonios de las y los jóvenes acerca de si se les motivaba para ir a la universidad, excepto en un caso que afirmó en el recurso le motivaban para que "estudie carrera, que no me vaya por las ramas, que me deje de CIP, que me deje de tonterías, que vaya a estudiar algo porque está chungo y hay que estudiar» (joven, mujer), en todos los demás casos, fue un no.

«noooo, ¡lo de la universidad olvídate! ¡Eso lo tenemos como súper... ¡porque en ningún momento se te ofrece ninguna ayuda para hacer la universidad! O sea, jeso lo tenemos súper claro! Y bachiller... depende, bueno las particulares y así sí te las pagan» (joven, mujer).

Entre los argumentos de las y los jóvenes también se hizo alusión a la edad de los 18 años como una edad de un antes y después en la intervención de los equipos, que pudiera determinar en cierto modo, las expectativas del equipo educativo, y las suyas propias. 
«ellos querían ver lo más fácil, ¿sabes? No sé, en plan de... ¡ten un trabajo ya! ¿Quién te dice que yo quiera trabajar ahora, tío? O no sé, es como que, tú a los 18 años no tienes a nadie y al final, tú tampoco sabes cómo voy a acabar en el piso, también nos decían: jigual no hay plazas, no sé qué! no hay plazas igual en el piso de mayores. Muchas veces esos miedos, ¿sabes?» (joven, hombre)

\subsection{La detección a través de la relación con jóvenes}

Al contrario de lo que ocurre con la mayoría de profesionales, la relación con las y los jóvenes no cesó los días de las entrevistas. Los mensajes de WhatsApp, los cafés, los encuentros y la relación ha ido estructurándose y convirtiéndose, en algunos casos más, en otros casos menos, en algo más que una relación entre personas investigadoras y entrevistadas. En los mencionados cafés y encuentros pudimos percibir la dificultad que tenían estas jóvenes (hablamos en femenino porque todas ellas son chicas) para poder realizar unos estudios superiores en comparación con la mayoría del alumnado universitario, principalmente, por falta de recursos económicos futuros. Las chicas que estaban cursando estudios universitarios vivían en un recurso residencial de inserción social y desde éste, se les ayudaba a costear gastos derivados de la universidad, pero, ¿cómo iban a hace frente a esos gastos cuando dejasen el recurso de inserción?, ¿cómo iban a costearse un máster profesionalizante necesario para ejercer su profesión?

No conocemos el dato exacto de cuál es la estancia media de jóvenes en recursos de inserción, aunque sabemos que pueden prorrogar su estancia hasta los 23 años ${ }^{3}$. No obstante, por las y los jóvenes entrevistados sabemos, que la estancia media rondaría el año y medio y dos años. Como alternativa a un recurso residencial de inserción o, después de la salida de estos, las y los jóvenes de entre 18 y 23 años pueden solicitar una prestación económica para jóvenes de la DFG ${ }^{4}$ que contempla una cuantía máxima de 700 euros. Esta cuantía contempla todos los gastos como el alquiler, la manutención, estudios, etc. ¿cómo hacer frente a un itinerario universitario?

\section{Procedimiento}

Con esta preocupación y con el objetivo de facilitar a estas jóvenes que ya estaban en la universidad o tenían intención de matricularse el curso académico siguiente el desarrollo de sus estudios universitarios, realizamos distintos contactos y expusimos al Vicerrectorado de Innovación y Compromiso Social y Acción Cultural la situación detectada, siendo, la consideración de colectivo exento de tasas el objetivo principal de nuestra demanda. Haciendo uso de unas pocas líneas de la demanda remitida, dejamos constancia de la dificultad y la desigualdad de oportunidades en las que se encuentran estas jóvenes:

En el caso de estas jóvenes, saben que no pueden volver a vivir con sus familiares ni cuentan con los medios económicos y afectivos que las demás personas cuentan. Entre los resultados también nos hemos percatado de que, como personas que les hubiera gustado ir a la universidad, han desistido, sea por incertidumbre, necesidad de trabajar o la duración de estos estudios, optando mayormente por otro tipo de itinerarios profesionalizantes. Con todo lo anteriormente relatado, y conociendo cuáles son los discursos profesionales y de jóvenes, opinamos que, apostando y ayudando a que las chicas y chicos extutelados, sin apoyos familiares y económicos a continuar sus estudios, la intervención profesional y las aspiraciones de las chicas y chicos tutelados y extutelados daría un cambio, siendo la universidad, también, una opción en su itinerario educativo y no sólo una utopía. Nos parece muy positivo que la Universidad traslade a las y los profesionales y personas que se encuentran en esta situación, que el acceso a la universidad y la obtención de estudios universitarios es una opción posible en su horizonte personal y profesional. Es cierto que el nivel educativo de las chicas y chicos tutelados en acogimiento residencial no es muy alto y que los casos presentados pueden ser casos extraordinarios, pero no por ello

\footnotetext{
${ }^{3}$ Más información http://www.cruzroja.es/principal/web/provincial-gipuzkoa/infancia-y-jovenes. Y http://koloreguztiak.org/..

${ }^{4}$ Ver más: Decreto Foral 5/2014 por la que se regula el procedimiento de acceso a recursos de inclusión social de atención secundaria dependientes de la DFG.
} 
hay que obviarlos, sino ayudar a que estas personas en desigualdad de condiciones tengan las mismas oportunidades que el resto de la población general. Existen grupos considerados «especialmente vulnerables» pero, acaso, ¿estas chicas y chicos no lo son?

Aunque fuimos percatándonos de las dificultades con las que contaban a lo largo de las transcripciones y posteriores análisis, la demanda tuvo que ser acelerada por el retraso en la resolución de la beca de estudios universitarios del Gobierno Vasco. La situación se condujo rápidamente, pero puso en evidencia la situación de desigualdad de oportunidades que padecen ya que:

- Son chicas que, por distintos motivos, son conscientes de que no pueden vivir con sus familias

- No tienen apoyos económicos que les puedan garantizar una continuidad en los estudios universitarios hasta la finalización de estos

- Actualmente viven en pisos de inserción o están percibiendo la prestación económica para jóvenes, pero no saben si van a poder finalizar sus estudios en estos pisos o con la ayuda

- Cuando salgan de los pisos de inserción van a tener la necesidad de trabajar un número elevado de horas semanales para poder afrontar los gastos de una vivienda, alimentación, transporte, estudios, etc., lo que dificultará y hará peligrar, la finalización de sus estudios.

Al tomar conciencia de la naturaleza del problema, un pequeño grupo de personas nos pusimos a trabajar en el tema, analizando las diferentes situaciones detectadas, examinando los recursos de apoyo con los que contaba la UPV/EHU para darles apoyo y, sobre todo, comprendiendo que esta situación iba mucho más allá de la solución de un problema puntual. Este fue el detonante de la solicitud de contacto con responsables de la DFG, que se materializó en una entrevista entre distintos cargos de la UPV/EHU y la DFG.

Esta entrevista dio como fruto una lista inicial de ideas que han sido re-elaboradas entre varias personas de las dos instituciones, así como por un pequeño grupo de jóvenes que podían acogerse en un futuro a este programa. Todas estas ideas buscaban en esencia garantizar el objetivo principal del programa: fortalecer y/o crear condiciones para favorecer el éxito académico universitario de jóvenes que están o que hayan llegado a su mayoría de edad bajo medidas de sistema de protección a la infancia y la adolescencia y/o participan en un programa de acompañamiento en la de servicios de inclusión social de la DFG.

\section{Las necesidades mencionadas por las jóvenes, la base del programa}

El punto de partida de este programa no podía ser otro que un diagnóstico bien documentado de las necesidades concretas que sentían las personas que se encontraban en esta situación. Para la elaboración de este primer borrador se contó con la colaboración de cuatro jóvenes extuteladas que seguían itinerarios educativos superiores y/o universitarios. Las necesidades que estas jóvenes consideraban de mayor relevantes eran:

- Recurso residencial o una alternativa habitacional durante todo el itinerario. Se trata sin lugar a dudas de la preocupación principal dado que estas jóvenes viven en una total incertidumbre sobre su futuro. Debemos tener en cuenta que un grado universitario es un itinerario de, al menos cuatro años, y que para ser exitoso se necesitan unas condiciones mínimas para el estudio.

- Recursos económicos básicos para hacer frente a: matrícula del grado, materiales de estudio, transporte, formaciones como cursos, seminarios, etc., e imprevistos.

- Acompañamiento emocional y académico, sobre todo en términos de orientación para la toma de decisiones y apoyo y feed-back en cuestiones relacionadas con los estudios.

\section{Coordinación interinstitucional}

Las necesidades demandadas por las jóvenes y su satisfacción necesitaban de la coordinación a varios niveles de distintos agentes: la UPV/EHU (personal académico y personal de servicios técnicos de atención al alumnado), la DFG (red los servicios sociales de atención secundaria, red de servicios sociales de protección a la infancia y la adolescencia) y las personas que vayan a tomar parte. 


\subsection{Desde las y los jóvenes que participen en el programa}

Por su parte, las chicas y chicos que se acojan al programa deberán adquirir ciertos compromisos y responsabilidades que garanticen el aprovechamiento en los estudios y que, a su vez, tomen parte activa en el mismo. Entre los compromisos y responsabilidades, destacaríamos las siguientes:

- Seguimiento académico trimestral tanto de la DFG como de la UPV.

- Compromiso de cumplir lo acordado con las obligaciones de los programas/recursos de inserción o protección.

- Matriculación mínima de un número determinado de créditos (según el caso, el grado y el curso).

- Superación de un mínimo las asignaturas (personalizadas en cada caso, según la dificultad del grado universitario).

\subsection{Desde la UPV/EHU}

La universidad consideraba que debía tomar parte, desde el primer momento que un chico o chica con el perfil anteriormente mencionado iría a matricularse en la UPV/EHU, creando un programa de acogida y seguimiento. Éste ofrecería dos tipos de apoyos, uno logístico y otro académico.

En cuanto al apoyo logístico, la universidad ha nombrado a una persona del Servicio de Orientación Universitaria (SOU) responsable de este cometido. Desde el primer momento en que una o un joven con las características anteriormente mencionadas se matricule en la UPV/EHU, la profesional del SOU, será la persona de referencia que les guie por el entramado de servicios y facilite los trámites administrativos de la Universidad. A su vez, recogerá las demandas y necesidades de las y los jóvenes y tramitará lo necesario para satisfacerlas (bonos de comida, material escolar, resolución de las becas del Gobierno Vasco, etc.).

En cuanto al apoyo académico, las chicas y chicos podrán contar con una persona del profesorado como referente durante su itinerario educativo mediante un programa de acompañamiento. En primer momento, se le asignaría un profesor o profesora provisional, pero después, las chicas o chicos podrán proponer quién quiere que sea su tutora o tutor académico. La persona que asuma la responsabilidad académica será un profesor o profesora de la titulación en la que la persona estudiante se haya matriculado y ejercerá esta tarea a lo largo de todo el grado.

Por otra parte, el mes de diciembre de 2017, el Gobierno de Rectorado creó un fondo económico para ayudar puntualmente en los gastos derivados de los estudios universitarios (traslado intercampus, manutención, material) ${ }^{5}$.

\subsection{Desde la DFG}

De acuerdo con el objetivo principal del programa, es imprescindible la colaboración y el trabajo en red con los Servicios Sociales de Atención Secundaria y los Servicios de Protección a la Infancia y la Adolescencia. Desde la red de protección a la infancia y la adolescencia se realizará una detección temprana de los itinerarios educativos universitarios, mediante una recogida sistemática de los datos del ámbito educativo de las y los adolescentes atendidos a partir de los 16 años. Resulta necesaria, en primera instancia, la detección temprana de jóvenes con potencial académico con objeto de abrir la posibilidad de desarrollar estudios a nivel universitario.

\footnotetext{
${ }^{5}$ En abril de 2018 la Fundación SM y la UPV/EHU han firmado un convenio para tres años por el cual la Fundación va a financiar parte de los gastos de los NNA implicados en el Programa Arrakasta de la UPV/EHU.
} 


\subsection{Desde la red de servicios sociales de atención secundaria de inclusión social}

Desde la red de servicios sociales de atención secundaria de inclusión social se trabajará a dos niveles. Por una parte, se realizará una acogida y seguimiento en el itinerario de educación superior de las y los jóvenes, ofreciendo a su vez, garantías que les permitirán cursan sus estudios de un modo exitoso (Alargar el tiempo de contrato de estancia de jóvenes que estén cursando estudios superiores, prorrogable hasta los 23 años; incluir una cuantía económica que permita a las chicas y chicos poder asumir los gastos derivados de los estudios, de manera complementaria a las becas del Gobierno Vasco, etc.). A su vez, en el caso de jóvenes que han estado bajo la medida de protección del acogimiento familiar, se les garantiza el mantenimiento de la ayuda económica del programa de emancipación en acogimiento familiar durante todo el itinerario formativo, limitado hasta los 23 años, al igual, que se les prestará apoyo psicológico en caso de necesidad, seguimiento periódico del proceso personal y formativo, acceso en determinadas circunstancias a ayudas complementarias para cubrir necesidades que se consideren básicas, entre otras.

Con la intención de darlo a conocer de forma específica al colectivo implicado estas dos instituciones se han comprometido a realizar reuniones divulgativas dentro las Redes de la DFG.

Para el cumplimiento y seguimiento técnico del programa Arrakasta se ha creado una Comisión de Seguimiento, formado por miembros de las dos instituciones. La evaluación del mismo se realizará anualmente.

\section{Primeras percepciones de jóvenes que están participando en el programa}

Para conocer las primeras percepciones acerca del programa pionero Arrakasta entrevistamos a cuatro de las seis jóvenes que actualmente participaban en él. El guion de la entrevista estuvo delimitado por cuatro preguntas, que hacían referencia, principalmente, a sus percepciones acerca de lo que este programa les ofrecía, y de la incidencia que podía tener o reportaba éste programa en otras personas. Debido a la disponibilidad de las participantes, en dos casos realizamos la entrevista de un modo presencial, y en otros dos, vía email.

En cuanto a las respuestas acerca de qué creían las jóvenes que aportaba el programa Arrakasta, responden que "aporta muchas cosas» (Iciar ${ }^{6}$ ) y que era un programa "totalmente necesario» (Sandra).

Entre las aportaciones que ellas perciben que les reportan este programa para cursar su itinerario educativo destacan «la tranquilidad» (Iciar) «la seguridad» (Sandra), "la visibilización de su colectivo» en la universidad (Lucía), «el apoyo y orientación en la universidad, saber a dónde ir» (Sandra), «la ayuda de material y de bonos de comida» (Sandra) e igualdad de oportunidades.

"que las y los jóvenes que salimos de centros de menores y que, por eso, tenemos muchas limitaciones (sobre todo económicas) podamos realizar una formación universitaria como todos los demás estudiantes que no cuentan con estas dificultades, es decir, que contemos con todo el material escolar (libros, fotocopias, ordenadores...) y que podamos contar con los servicios de la universidad como el comedor universitario si lo necesitamos» (María).

En cuanto a su percepción sobre si este programa va a reportar a otras personas y/o va a incidir en otros contextos, las jóvenes destacan tres aspectos claves, aunque opinan que este programa «puede transmitir muchísimo a muchísima gente» (Iciar). El primero de los aspectos que destacan es, que va a posibilitar e impulsar a otras NNA en AR a cursar estudios universitarios. Como ellas mismas relataban,

"supone un pequeño grano de esperanza para personas tuteladas que tengan en mente cursar estudios universitarios» (Lucía).

"La idea de que vas a poder, de que hay algo más adelante (...) a mí me parece muy importante que, desde muy pequeña, por ejemplo, a otras personas que se sepa que no está sólo el CIP, pues eso, o incluso grado medio o superior, que no me parece mal, que ni lo infravaloro

${ }^{6}$ Todos los nombres expuestos son seudónimos. 
ni nada, pero sí me parece importante tener en cuenta que también puedes llegar a la universidad y Arrakasta ayuda muchísimo para eso» (Iciar).

El segundo aspecto destacado es que este programa también tendrá reflejo en las orientaciones educativas que los equipos educativos de AR sugieren a las NNA o éstas y éstos se sientan, de cierta manera, obligados a decantarse por ciertos itinerarios educativos, por ese yugo de la mayoría de edad. Sandra lo relataba del siguiente modo,

«que muchos jóvenes que se esfuerzan en sus estudios y les gustaría continuar con su formación al cumplir los 18 años consigan acceder a la universidad y que, de este modo, no se vean obligados a buscar un trabajo rápidamente y olvidarse de su educación».

El tercer aspecto es que perciben que este programa puede ayudar a cambiar miradas en la sociedad, a deconstruir imágenes sociales, principalmente negativas asociadas a las NNA en AR.

"con Arrakasta se visibiliza que hay gente que llega más o que, hay gente que está estudiando en la universidad o lo que sea, y que no sólo son personas que no hacen nada, que son delincuentes» (Iciar).

Se realizaron dos propuestas de mejora para el Departamento de Educación del Gobierno Vasco, la ampliación de este programa a jóvenes que estudian estudios superiores, aunque no universitarios, y la declaración de colectivo exento de tasas por ser un colectivo en desigualdad de condiciones.

"Yo creo que habría que ampliarlo más, habría que abrirlo a educación superior por lo menos, a mí sí me parece, por lo menos. Porque igual hay mucha gente que no quiere llegar a la universidad y, ¿por qué esa gente no tiene derecho a tener una ayuda de una forma especial? Porque son también residentes de piso, son también... no sólo es éxito porque llegues a la universidad, sino que hay muchos más casos de éxito. ¡Éxito tampoco es universidad, y ya! ¡O estudios y ya! El éxito son muchísimas cosas» (Iciar).

"a mí me falta el Gobierno Vasco y las becas, deberían considerarnos como especial, especial vulnerabilidad, las palabras que quieras poner, pero deberían considerarnos como un colectivo que necesita discriminación positiva. Si así fuera yo podría hacer un máster» (Lucía).

\section{7.- A modo de cierre...}

En líneas anteriores hemos expuesto, a grandes rasgos, un programa pionero que tiene como objetivo «blindar» a jóvenes que han egresado del sistema de protección para que puedan realizar su itinerario educativo universitario de un modo exitoso.

Como hemos indicado al comienzo de la comunicación, las NNA en AR suelen tener un bajo nivel formativo y un porcentaje no muy alto suele llegar a realizar estudios universitarios. Detrás de dichas afirmaciones hay muchas y muy diversas razones en las cuales no vamos a explayarnos en esta comunicación, pero que nos invitan a reflexionar y a replantearnos nuestra responsabilidad social sobre la adaptabilidad de las políticas educativas y sociales en la cobertura de las necesidades de estas jóvenes. Esta responsabilidad no es tarea de una institución y/o de un grupo de profesionales, es una tarea común, que necesita de la coordinación y la colaboración de distintos agentes que remen en la misma dirección. El reconocimiento del Gobierno Vasco al colectivo formado por jóvenes procedentes del sistema de protección como colectivo en desigualdad de condiciones, sería un buen aporte a esta reflexión y a este programa.

Las primeras percepciones del programa indican que va más allá del «blindaje» de los itinerarios universitarios. Arrakasta crea esperanza, rompe límites y cambia miradas y horizontes de distintos agentes y puede ser un programa modelo para ser aplicado en otros contextos, ya que contribuyen a la mejora del derecho a la educación. 


\section{REFERENCIAS BIBLIOGRÁFICAS}

Beiter, K. (2005). The Protection of the Right to Education by International Law. Boston: Martinus Nijhoff publisher.

Casas, F., y Montserrat, C. (2012). Percepciones de distintos agentes sociales acerca de la educación formal de los chicos y chicas tutelados. Educatio Siglo XXI, 30(2), 185-206. Recuperado de https://www.siis.net/documentos/ficha/206572.pdf.

Cashmore, J. Paxman, M. y Townsend, M. (2007). The educational outcomes of young people 4-5 years after leaving care An Australian perspective. Adoption \& fostering, 31(1), 50-62 http://journals.sagepub.com/doi/pdf/10.1177/030857590 703100109.

Federació d'Entitats amb Projectes i Pisos Assistits (FEPA) (2017). Memòria 2016. Barcelona: FEPA i Diputació de Barcelona. Recuperado de https://www.fepa18.org/wpcontent/uploads/2013/10/Memoria_FEPA-cat2016.pdf.

Harvey, A., McNamara, P., Andrewartha, L., \& Luckman, M. (2015). Out of care, into university: Raising higher education access and achievement of care leavers. Perth, Australia: National Centre for Student Equity in Higher Education (NCSEHE), Curtin University. Recuperado de https://www.ncsehe.edu.au/wp-content/uploads/2015/03/Out-of-Care-Into-University.pdf.

Jackson, S., y Martin, P. Y. (1998). Surviving the care system: education and resilience. Journal of adolescence, 21(5), 569-583.

Jackson, S. y Martin, P. (2002). Educational success for children in public care: advice from a group of high achievers. Child and Family Social Work, 7, 121-130. Recuperado de https://onlinelibrary.wiley.com/doi/epdf/10.1046/j.13652206.2002.00240.x.

Melendro et al. (2016) Jóvenes sin tiempo: Riesgos y oportunidades de los jóvenes extutelados en el tránsito a la vida adulta. Recuperado de https://www.researchgate.net/publication/320595864_Jovenes_sin_tiempo_Riesgos_y_oportunidades_de_los_jovenes_extutelados_en_el_transito_a_la_vida_adulta_Investigacion_Young_people_without_ time_Risks_and_opportunities_of_the_extutelated_youth_into_the_.

Mallon, J. (2007). Returning to education after care: protective factors in the development of resilience. Adoption \& Fostering, 31(1), 106-117.

Montserrat, C y Casas, F. (2010). Educación de jóvenes extutelados: Revisión de la literatura científica española. Educatio Siglo XX1, 13(2), 117-138. Recuperado de http://revistas.uned.es/index.php/educacionXX1/article/view/240/198.

Montserrat, C., Casas, F. y Baena, M. (2015). La educación de niños, niñas y adolescentes en el sistema de protección ¿Un problema o una oportunidad? Girona: IRQV y Documenta Universitaria.

Montserrat, C. et al. (2011). Los itinerarios educativos de los jóvenes ex-tutelados. Informes, estudios e investigación 2011. Ministerio de Sanidad, Política social e Igualdad. Recuperado de http://www.observatoriodelainfancia.msssi.gob.es/ productos/pdf/UCl2011.pdf.

Panchón (dir.) (1999). Situación de menores de 16 a 18 años en centros de protección. Disponible en: http://www.dulac.org/ PDFs/resinvmenores.pdf.

Tomaševski, K. (2004). El asalto a la Educación. Barcelona: Intermon Oxfam.

Tomaševski, K. (2005). «El derecho a la educación, panorama internacional de un derecho irrenunciable». En L. M. Naya (ed.). La educación y los derechos humanos (pp. 63-90). Donostia: Erein.

Tomaševski, K. (2006). Human Rights Obligations in Education. The 4-As Scheme. Nijmegen: Wolf Legal Publishers.

UNESCO (1960). Convención de la UNESCO relativa a la lucha contra la discriminación en la esfera de la enseñanza. Recuperado de http://portal.unesco.org/es/ev.php-URL_ID=12949\&URL_DO=DO_TOPIC\&URL_SECTION=201.html.

UNESCO (1988). Declaración Mundial sobre la Educación Superior en el siglo XXI: visión y acción, aprobada por la Conferencia Mundial sobre la Educación Superior el 9 de octubre de 1998 en la sede de la UNESCO en París. Recuperado de http://unesdoc.unesco.org/images/0011/001163/116345s.pdf. 